\title{
Expression of Gamma-Glutamyltransferase(s) and Intracellular Glutathione Levels: An Open Issue
}

\section{Corti A* and Pompella A}

Department of Translational Research and New

Technologies in Medicine and Surgery, University of Pisa, Italy

*Corresponding author: Corti A, Department of Translational Research and New Technologies in Medicine and Surgery, Medical School, University of Pisa, 56126 Pisa, Italy

Received: January 20, 2017; Accepted: February 17, 2017; Published: February 20, 2017

\section{Keywords}

Gamma-glutamyltransferase; Glutathione; Redox regulation

\section{Perspective}

Gamma-Glutamyltransferase (GGT) is the enzyme known to be involved in the metabolism of the major antioxidant glutathione ( $\gamma$-Glu-Cys-Gly; GSH). GGT is also one of the most used clinical markers of liver damage and its activity is routinely analyzed in blood or plasma [1].

GGT is widely distributed in living organisms and in humans at least eight GGT genes or pseudogenes have been identified [2]. Among the others, GGT1 and GGT5 are the only two members of GGT family that produce a functional protein [2]. In particular, both enzymes are able to hydrolyze the gamma-glutamyl bond of glutathione and glutathione S-conjugates and catalyze the transfer of gamma-glutamyl groups between different compounds (i.e. hydrolysis and transpeptidation) [3], even if with different substrate specificity. Under physiological conditions, the predominant reaction catalyzed by GGT is supposed to be the hydrolysis of gamma-glutamyl bond [3]. For both GGT1 and GGT5, the mature enzyme is a membrane bound glycoprotein which consists of two polypeptide chains, i.e. a light and a heavy subunit [2].

GGT1 is the most studied and most ubiquitously expressed human GGT gene, but the crystal structure of human GGT1 has been only recently described [4]. A dysregulated expression of GGT1 has been detected in several tumor types and its role in tumor progression and drug resistance has been extensively investigated [5]. GGT1 catalyzes the degradation of extracellular GSH, thus favouring the recovery of constituent amino acids and contributing to the modulation of cysteine homeostasis and redox status of cells. Playing a role in the sequence of reactions forming the so-called "gamma-glutamyl cycle", GGT has been early recognized as a component of the cell protection system against oxidative stress [1]. However, a number of findings indicate that, under selected conditions, the metabolism of GSH by GGT can produce pro-oxidant effects and can be implicated in the modulation of several redox-sensitive processes, possibly contributing to tumor progression [6]. These effects are mediated by cysteinyl-glycine - the first product of GSH catabolism mediated by
GGT - which is able to reduce extracellular transition metal cations $\left(\mathrm{Fe}^{+}, \mathrm{Cu} 2^{+}\right)$more efficiently than GSH, thus leading to the formation of superoxide anion, hydrogen peroxide and thiyl radicals [5].

Apart from reduced glutathione, other gamma-glutamyl compounds are GGT1 substrates, e.g. oxidized glutathione (GSSG), pathophysiologically relevant compounds such as the adducts GSHxenobiotics produced by glutathione-S-transferases [7] and two of the major inflammatory mediators, namely leukotriene C4 $[8,9]$ and S-nitroso-glutathione [10]. In addition, also synthetic gammaglutamyl compounds are substrates for GGT1 and the developement of gamma-glutamyl anticancer pro-drugs - that can be selectively cleaved by GGT expressed by cancer cells - has been taken into account $[11,12]$. The transcriptional regulation of GGT1 gene is quite complex [13] and its expression may be promoted by both oxidative and inflammatory stimuli. As other enzymes involved in the GSH metabolism, GGT can be upregulated by compounds producing an increase in Reactive Oxygen/Nitrogen Species (ROS/RNS) [14-19].

GGT5 is the only other member of the GGT family provided with catalytic activity. TC4 was the first recognized substrate for GGT5 (previously named Gamma-Glutamyl-Leukotrienase (GGL; [20]), whereas GSH and GSSG were suggested to be substrates for GGT5 in humans [2,3] but not in mice [20]. However, as judged by the rate of glutamate release from GSH, GGT5 cleaves GSH approximately 46 times slower than GGT1 [3] and it is not able to cleave GammaGlutamyl p-Nitroanalide (GpNA), a nonphysiologic compound commonly used to assay GGT1 activity in biological samples [21]. Thus GG1 and GGT5 are both able to hydrolyze the gamma-glutamyl bond but they have different-only partially overlapping-substrate specificity. GGT5 localization was only recently deeply studied and revealed that GGT5 is expressed in some of the same tissues expressing GGT1, but in different subsets of cells. It has been proposed that this localization may result in different access to substrates, i.e. blood and intercellular fluids for GGT5 and fluids in ducts and glands for GGT1 [22]. On the other hand, little is currently known about the regulation of GGT5 expression.

What is the real physiological significance of enzymatically active GGT's? A s stated above, both GGT1 and GGT5 - even though with different efficiencies - play a unique and specific role in the catabolism of GSH, being the sole enzymes which are able to hydrolyze the gamma-glutamyl bond, thus allowing the recovery of cysteine for intracellular GSH synthesis. However, conflicting results were reported about this specific point. No difference or even a decrease rather than an increase - of intracellular GSH was repeatedly detected in different cancer cell lines upon transfection with GGT1 cDNA, both in vitro and after transplantation in nude mice [23-26]. These apparent inconsistencies can be at least in part explained taking into account the pro-oxidant effects produced by the GGT1-meditated GSH catabolism in the presence of extracellular metal ions [27] and 
the involvement of GGT1 in S-cysteylglycylation reactions [28].

Interestingly, similar results have been now also reported in a recent paper on GGT5 in mice [29]. As for human specimens [22], GGT5 is expressed by the interstitial Leydig cells in the mouse testis. Insulin-resistent diabetic mice are characterized by low levels of testosterone and increased expression of GGT5 in testes. In vitro studies demonstrated that the induced over expression of GGT5 by plasmid transfection in the TM3 Leydig cell line was associated with a significant reduction of intracellular GSH levels, increased expression of heme oxygenases-1 and reduced amounts of cytochrome P450 [29]. Upon stimulation of steroidogenesis by Luteinizing Hormone (LH), GGT5 over expressing cells showed higher levels of Malondialdehyde (MDA) and a reduced production of testosterone, as compared to the corresponding control. These effects are supposed to be mediated through the lower levels of GSH associated with GGT5 over expression, making cells more vulnerable to the reactive oxygen species produced during steroidogenesis [29].

These findings suggest that the over expression of GGT1 and/ or GGT5 may produce similar effects on intracellular GSH levels, both contributing to the modulation of the redox state of the cells in a pro oxidant direction. It is well known that tissue culture media used for in vitro experiments could affect the levels of intracellular GSH, depending on their cysteine levels. However, conflicting results about GGT1 expression and intracellular GSH levels were obtained from in vivo studies by the comparison of GGT-positive VS. GGT-negative tumors obtained after injection into nude mice of human prostate carcinoma [24] or human melanoma [26] cells transfected with the full length human GGT1 cDNA. Indeed, over expression of GGT provided a growth advantage to tumor cells in vivo, but the glutathione levels in the GGT-positive tumors were similar [24] or even significantly lower [26] as compared to those detectable in the GGT-negative tumors. Against this background, the traditional characterization of GGT enzymes as members of the cellular 'antioxidant' machineries seems no more warranted, and data reported in the literature from several independent laboratories point rather to a more complex involvement in cellular redox homeostasis of these activities.

A better understanding of GGT1 and GGT5 expression/ functions (as well as of the factors possibly modulating their action in cells and tissues) could provide a significant improvement in our comprehension of their role(s) in cellular redox-sensitive signalling, and likely of the real significance of GGT levels in serum and other body fluids.

\section{References}

1. Whitfield JB. Gamma glutamyl transferase. Crit Rev Clin Lab Sci. 2001; 38 263-355.

2. Heisterkamp N, Groffen J, Warburton D, Sneddon TP. The human gammaglutamyltransferase gene family. Hum Genet. 2008; 123: 321-332.

3. Wickham S, West MB, Cook PF, Hanigan MH. Gamma-glutamyl compounds: substrate specificity of gamma-glutamyl transpeptidase enzymes. Anal Biochem. 2011; 414: 208-214.

4. West MB, Chen Y, Wickham S, Heroux A, Cahill K, Hanigan MH, et al. Nove insights into eukaryotic $Y$-glutamyltranspeptidase 1 from the crystal structure of the glutamate-bound human enzyme. J Biol Chem. 2013; 288: $31902-$ 31913.
5. Corti A, Franzini M, Paolicchi A, Pompella A. Gamma-glutamyltransferase of cancer cells at the crossroads of tumor progression, drug resistance and drug targeting. Anticancer Res. 2010; 30: 1169-1181.

6. Corti A, Duarte TL, Giommarelli C, De Tata V, Paolicchi A, Jones GD, et al. Membrane gamma-glutamyl transferase activity promotes iron-dependent oxidative DNA damage in melanoma cells. Mutat Res. 2009; 669: 112-121.

7. Tate SS, Meister A. Gamma-Glutamyl transpeptidase: catalytic, structural and functional aspects. Mol Cell Biochem. 1981; 39: 357-368.

8. Lewis RA, Austen KF, Soberman RJ. Leukotrienes and other products of the 5-lipoxygenase pathway. Biochemistry and relation to pathobiology in human diseases. N Engl J Med. 1990; 323: 645-655.

9. Mayatepek E, Okun JG, Meissner T, Assmann B, Hammond J, Zschocke $\mathrm{J}$, et al. Synthesis and metabolism of leukotrienes in gamma-glutamyl transpeptidase deficiency. J Lipid Res. 2004; 45: 900-904

10. Hogg N, Singh RJ, Konorev E, Joseph J, Kalyanaraman B. S-Nitrosoglutathione as a substrate for gamma-glutamyl transpeptidase. Biochem J. 1997; 323: 477-481.

11. Dilda PJ, Ramsay EE, Corti A, Pompella A, Hogg PJ. Metabolism of the tumor angiogenesis inhibitor 4-(N-(S-Glutathionylacetyl)amino)phenylarsonous acid. J Biol Chem. 2008; 283: 35428-35434.

12. Ramsay EE, Decollogne S, Joshi S, Corti A, Apte M, Pompella A, et al. Employing pancreatic tumor $\mathrm{y}$-glutamyltransferase for therapeutic delivery. Mol Pharm. 2014; 11: 1500-1511.

13. Ikeda Y, Taniguchi N. Gene expression of gamma-glutamyltranspeptidase. Methods Enzymol. 2005; 401: 408-425.

14. Kugelman A, Choy HA, Liu R, Shi MM, Gozal E, Forman HJ. gammaGlutamyl transpeptidase is increased by oxidative stress in rat alveolar L2 epithelial cells. Am J Respir Cell Mol Biol. 1994; 11: 586-592.

15. Ripple MO, Henry WF, Rago RP, Wilding G. Prooxidant-antioxidant shift induced by androgen treatment of human prostate carcinoma cells. J Natl Cancer Inst. 1997; 89: 40-48.

16. Choi J, Liu RM, Forman HJ. Adaptation to oxidative stress: quinone-mediated protection of signaling in rat lung epithelial L2 cells. Biochem Pharmacol. 1997; 53: 987-993.

17. Takahashi Y, Oakes SM, Williams MC, Takahashi S, Miura T, Joyce-Brady $M$. Nitrogen dioxide exposure activates gamma-glutamyl transferase gene expression in rat lung. Toxicol Appl Pharmacol. 1997; 143: 388-396.

18. Ravuri C, Svineng G, Pankiv S, Huseby NE. Endogenous production of reactive oxygen species by the NADPH oxidase complexes is a determinant of Y-glutamyltransferase expression. Free Radic Res. 2011; 45: 600-610.

19. Pandur S, Ravuri C, Moens U, Huseby NE. Combined incubation of colon carcinoma cells with phorbol ester and mitochondrial uncoupling agents results in synergic elevated reactive oxygen species levels and increased Y-glutamyltransferase expression. Mol Cell Biochem. 2014; 388: 149-156.

20. Carter BZ, Wiseman AL, Orkiszewski R, Ballard KD, Ou CN, Lieberman MW. Metabolism of leukotriene C4 in gamma-glutamyl transpeptidase-deficient mice. J Biol Chem. 1997; 272: 12305-12310.

21. Heisterkamp N, Rajpert-De Meyts E, Uribe L, Forman HJ, Groffen J. Identification of a human gamma-glutamyl cleaving enzyme related to, but distinct from, gamma-glutamyl transpeptidase. Proc Natl Acad Sci USA 1991; 88: 6303-6307.

22. Hanigan MH, Gillies EM, Wickham S, Wakeham N, Wirsig-Wiechmann CR. Immunolabeling of gamma-glutamyl transferase 5 in normal human tissues reveals that expression and localization differ from gamma-glutamyl transferase 1. Histochem Cell Biol. 2015; 143: 505-515.

23. Bailey HH, Gipp JJ, Mulcahy RT. Increased expression of gamma-glutamyl transpeptidase in transfected tumor cells and its relationship to drug sensitivity. Cancer Lett. 1994; 87: 163-170.

24. Hanigan MH, Gallagher BC, Townsend DM, Gabarra V. Gamma-glutamyl transpeptidase accelerates tumor growth and increases the resistance of tumors to cisplatin in vivo. Carcinogenesis. 1999; 20: 553-559. 
25. Karp DR, Shimooku K, Lipsky PE. Expression of gamma-glutamy transpeptidase protects ramos B cells from oxidation-induced cell death. $J$ Biol Chem. 2001; 276: 3798-3804

26. Franzini M, Corti A, Lorenzini E, Paolicchi A, Pompella A, De Cesare M, et al. Modulation of cell growth and cisplatin sensitivity by membrane gammaglutamyltransferase in melanoma cells. Eur J Cancer. 2006; 42: 2623-2630.

27. Pompella A, Corti A, Paolicchi A, Giommarelli C, Zunino F. Gammaglutamyltransferase, redox regulation and cancer drug resistance. Curr Opin Pharmacol. 2007; 7: 360-366.
28. Corti A, Paolicchi A, Franzini M, Dominici S, Casini AF, Pompella A. The S-thiolating activity of membrane gamma-glutamyltransferase: formation of cysteinyl-glycine mixed disulfides with cellular proteins and in the cell microenvironment. Antioxid Redox Signal. 2005; 7: 911-918.

29. Li W, Wu ZQ, Zhang S, Cao R, Zhao J, Sun ZJ, et al. Augmented expression of Gamma-Glutamyl Transferase 5 (GGT5) impairs testicular steroidogenesis by deregulating local oxidative stress. Cell Tissue Res. 2016; 366: 467-481.
Austin J Pathol Lab Med - Volume 4 Issue 1 - 2017

ISSN : 2471-0156 | www.austinpublishing group.com

Corti et al. () All rights are reserved
Citation: Corti A and Pompella A. Expression of Gamma-Glutamyltransferase(s) and Intracellular Glutathione Levels: An Open Issue. Austin J Pathol Lab Med. 2017; 4(1): 1018. 